\title{
Mucosal Immunization Induces a Higher Level of Lasting Neutralizing Antibody Response in Mice by a Replication-Competent Smallpox Vaccine: Vaccinia Tiantan Strain
}

\author{
Bin Lu, ${ }^{1}$ Wenbo Yu, ${ }^{2}$ Xiaoxing Huang, ${ }^{1}$ Haibo Wang, ${ }^{2}$ Li Liu, ${ }^{2}$ and Zhiwei Chen ${ }^{2,3}$ \\ ${ }^{1}$ AIDS Center and Modern Virology Research Center, State Key Laboratory of Virology, College of Life Sciences, \\ Wuhan University, Hubei 430072, China \\ ${ }^{2}$ AIDS Institute, Li Ka Shing Faculty of Medicine, The University of Hong Kong, Pokfulam, Hong Kong \\ ${ }^{3}$ Department of Microbiology and Research Center for Infection and Immunity, Li Ka Shing Faculty of Medicine, \\ The University of Hong Kong, Pokfulam, Hong Kong \\ Correspondence should be addressed to Zhiwei Chen, zchenai@hku.hk
}

Received 23 February 2011; Revised 18 April 2011; Accepted 19 April 2011

Academic Editor: Young-Chul Sung

Copyright () 2011 Bin Lu et al. This is an open access article distributed under the Creative Commons Attribution License, which permits unrestricted use, distribution, and reproduction in any medium, provided the original work is properly cited.

\begin{abstract}
The possible bioterrorism threat using the variola virus, the causative agent of smallpox, has promoted us to further investigate the immunogenicity profiles of existing vaccines. Here, we study for the first time the immunogenicity profile of a replicationcompetent smallpox vaccine (vaccinia Tiantan, VTT strain) for inducing neutralizing antibodies (Nabs) through mucosal vaccination, which is noninvasive and has a critical implication for massive vaccination programs. Four different routes of vaccination were tested in parallel including intramuscular (i.m.), intranasal (i.n.), oral (i.o.), and subcutaneous (s.c.) inoculations in mice. We found that one time vaccination with an optimal dose of VTT was able to induce anti-VTT Nabs via each of the four routes. Higher levels of antiviral Nabs, however, were induced via the i.n. and i.o. inoculations when compared with the i.m. and s.c. routes. Moreover, the i.n. and i.o. vaccinations also induced higher sustained levels of Nabs overtime, which conferred better protections against homologous or alternating mucosal routes of viral challenges six months post vaccination. The VTT-induced immunity via all four routes, however, was partially effective against the intramuscular viral challenge. Our data have implications for understanding the potential application of mucosal smallpox vaccination and for developing VTT-based vaccines to overcome preexisting antivaccinia immunity.
\end{abstract}

\section{Introduction}

Smallpox, which was caused by infection with the variola virus, was one of the most deadly diseases in human history with a mortality rate of up to $50 \%[1,2]$. Fortunately, this disease was completely eradicated throughout the world by 1980 after the introduction of a global smallpox vaccination campaign [3]. During this process, vaccinia virus, which shares broad antigenic properties with the variola virus, played an essential role as an effective vaccine in inducing protective immunity against smallpox [3-5]. It is well accepted that vaccine-induced neutralizing antibodies are critical to protection [6-8]. The fear of variola virus being deliberately released in potential bioterrorism attacks and the increasing use of vaccinia as vaccine vectors for other diseases such as AIDS have led to more recent studies aimed at understanding the protective immune responses induced by the smallpox vaccine $[9,10]$. It is, therefore, necessary to investigate the neutralizing antibody responses induced by historically used smallpox vaccines via different route of vaccination.

The most extensively used smallpox vaccine in China was the vaccinia virus Tiantan (VTT) strain [11]. Accordingly, the original VTT was isolated from the skin lesion of a smallpox patient in China around 1926 followed by extensive passages of 3 times in monkeys, 5 in rabbits, 3 in bovines, 
then twice in rabbits and 3 times in bovines, repeatedly [12, 13]. Genetic analysis of VTT genome, however, suggested that it is a vaccinia strain instead of a variola viral variant [14]. The clinical safety of this vaccine has not been clearly documented although VTT was historically used for millions of people. These issues are essential for a safe smallpox vaccine [15]. The biological characteristics of VTT have been described in our recent studies $[16,17]$. It was reported that VTT caused larger lesions after intradermal vaccination and was likely more virulent than other widely used smallpox vaccines such as Lister or Wyeth $[12,18]$. To date, whether or not VTT can induce protective neutralizing antibody responses through noninvasive mucosal vaccination remains less understood $[19,20]$.

Here, we study VTT to investigate its immunogenicity in terms of inducing neutralizing antibodies through four different routes of vaccination in a mouse model, which has not been previously studied. Moreover, by conducting homologous and heterologous routes of viral challenges, we aimed to determine the efficacy of VTT for protection and to identify a strategy to overcome preexisting immunity to VTT-based vaccines. This study involved a safe, nonpathogenic viral challenge model using a high dose of modified VTT, namely, MVTT-S, which expresses the spike (S) glycoprotein of SARS-CoV. Since $\mathrm{S}$ is not expressed on the surface of vaccinia virus, we aimed to determine the role of anti-VTT neutralizing antibody (Nab) responses in achieving protection by evaluating the seroconversion to $S$. Our results have implications for understanding an aspect of vaccinia-induced protective immunity and for developing vaccinia-based vaccines.

\section{Materials and Methods}

2.1. Virus Stock and Cell Line. The background and biological properties of the smallpox vaccine vaccinia Tiantan (VTT) have been described previously [16]. VTT stocks were propagated in Vero cells and then purified by repeated freezing and thawing and centrifugation through a 36\% sucrose cushion. The viral pellet was subjected to a sucrose gradient centrifugation. Purified viruses were collected and studied [21]. The plaque forming unit (PFU) of viral stocks was titrated on Vero cells by a plaque-forming assay using crystal violet staining. The construction and characterization of the challenge virus MVTT-S have been described previously [17].

2.2. Immunization of Mice. Five groups of female BALB/c mice were included in the study. Each group of nine mice was inoculated with an optimal dose of $10^{6}$ PFU VTT through one of four different routes including intramuscular (i.m.), intranasal (i.n.), oral (i.o.), and subcutaneous (s.c.) inoculations, respectively, [17]. Another group of nine mice received a placebo as controls. To measure lasting $\mathrm{Nab}$ responses, mice were kept for six months for observation and testing.

2.3. Viral Challenge of VTT-Immunized Mice. After six months of observation, each group of mice was further divided into three subgroups. Each subgroup of three mice was challenged with a dose of $2 \times 10^{6}$ PFU MVTT-S through one of three different routes including i.m., i.n., and i.o., respectively. It is the highest dose possible for mucosal challenge based on the concentration of our viral stocks and the size of animals. After a three-week interval, all animals were challenged again with the same dose of $2 \times 10^{6}$ PFU MVTT-S. Blood samples were collected on week 3, week 26, week 29, and week 32 for measuring Nab responses after MVTT-S challenge. Our animal protocols were approved by the Institutional Committee on Laboratory Animal Use of Wuhan University. Since MVTT-S is an attenuated VTT variant which is not lethal in mice, we define the full protection by the lack of detectable anti-S $\mathrm{Nab}$ response induced by the challenge virus MVTT-S in this study.

2.4. Anti-S Neutralizing Assay. A pseudovirus-based neutralization assay was applied to determine the humoral immune responses against the $\mathrm{S}$ glycoprotein of SARS-CoV [22]. The pseudotype virus was generated by cotransfecting 293T cells with two plasmids pcDNA-Sopt9 and pNL43Luc+Env-Vpr- carrying the optimized $\mathrm{S}$ gene and a human immunodeficiency virus type 1 backbone, respectively. For each experiment, pooled sera, which contain an equal amount of serum of each vaccinated mouse in each group, were subjected to the assay as described by others [23]. Luciferase activity was measured by the PerkinElmer Victor Microplate Reader according to manufacturer's instruction. In comparison to serum samples of unvaccinated mice in the same experiments, we score seropositive when an $\mathrm{IC}_{50}$ value reaches a serum dilution factor of $1: 30$. An $\mathrm{IC}_{50}$ value is a serum dilution factor that achieves $50 \%$ of viral inhibition.

2.5. Anti-VTT Neutralizing Assay. To test the serum neutralization activity against VTT, we used a flow-cytometrybased assay similar to a previously published method [24]. For this assay, a modified VTT that contains a GFP (MVTTGFP) expression cassette was constructed [14]. Briefly, the neutralizing activity of heat-inactivated sera $\left(56^{\circ} \mathrm{C}, 30 \mathrm{~min}\right)$ was determined by mixing $1.25 \times 10^{6} \mathrm{PFU} / \mathrm{mL}$ of VTTGFP virus in $20 \mu \mathrm{L}$ with an equal amount of diluted serum at $37^{\circ} \mathrm{C}$, for $1 \mathrm{~h}$. After the incubation, $60 \mu \mathrm{L}$ of the $293 \mathrm{~T}$ cells were added into each well of the plate and incubated at $37^{\circ} \mathrm{C}$. $293 \mathrm{~T}$ cells were diluted into $2.5 \times 10^{6} / \mathrm{mL}$ using culture medium containing 10\% FBS, 1\% streptomycin and penicillin, and $44 \mu \mathrm{g}$ of cytosine arabinoside per $\mathrm{mL}$. After $24 \mathrm{hr}$ incubation, the infected cells were washed with PBS twice and then trypsinized ( $50 \mu \mathrm{L} /$ well trypsin). The cells were pelleted, washed, and then fixed with $200 \mu \mathrm{L}$ of $2 \%$ paraformaldehyde, and subsequently subjected to the FACS analysis. The titer of viral neutralization was determined by calculating the percent reduction of infected cells showing GFP positive expression. Similarly, an $\mathrm{IC}_{50}$ value is a serum dilution factor that achieves $50 \%$ of viral inhibition. 
2.6. Statistical Analyses. The Student's $t$-test was used to evaluate the statistical significance of $\mathrm{Nab}$ titers among various groups of animals. The Origin 8 computer software was used for this analysis.

\section{Results}

3.1. Higher Levels of Anti-VTT Nabs Were Induced via the i.n. and i.o. Inoculations When Compared with the i.m. and s.c. Routes. In this study, we sought to determine whether VTT would induce different levels of Nabs via various routes of vaccination. This is a critical question because VTT was historically used as a smallpox vaccine for millions of people yet its potential use as a noninvasive vaccine remains elusive. To address this question, four groups of mice were inoculated with an optimal dose of $10^{6} \mathrm{PFU}$ VTT via s.c, i.m., i.n., and i.o. routes, respectively. Another group of mice received a placebo as controls. Three weeks after inoculation, the animals were tested for the production of anti-VTT Nabs using a newly developed FACS-based MVTT-GFP neutralization assay (Figure 1(a)) [24]. Since the sample quantity is small, pooled sera were tested for each experimental group. The pooled sera contain an equal amount of serum of individualmice in each group. We found that each route of vaccination was able to induce anti-VTT $\mathrm{Nab}$ responses after one-time vaccination (Figure $1(\mathrm{~b})$ ). The levels of anti-VTT Nabs, however, varied depending on the route of vaccination. Apparently, higher levels of Nabs were induced via the i.n. and i.o. inoculations when compared with the i.m. and s.c. routes. The serum dilution factors based on the $\mathrm{IC}_{50}$ value via the different routes (i.n., i.o., i.m., and s.c.) were $1: 711,1: 2370,1: 428$, and $1: 32$, respectively.

\subsection{Mucosal Vaccination Also Induced Higher Sustained Levels} of Nabs over time. In order to measure the long-lasting levels of Nabs generated, we kept separate groups of the vaccinated animals for six months. Despite the virulent effects of VTT (e.g., body weight changes) during the acute phase of vaccination, all mice survived for continuous observation. After six months of the vaccination, serum samples were collected and subsequently subjected to the same neutralization assay. Interestingly, although the $\mathrm{IC}_{50}$ was not significantly improved for the i.o. group, the rest of three groups of mice, especially the i.n. group, developed higher levels of lasting antivaccinia Nabs when compared to the early time point (Figures $1(\mathrm{~b})$ and $1(\mathrm{c})$ ), suggesting a prolonged VTT-antigen stimulation to immune system. The serum dilution factors based on the $\mathrm{IC}_{50}$ value via the different routes (i.n. and i.o. versus i.m. and s.c.) were $1: 2265$ and $1: 2397$ (average $1: 2331$ ) versus $1: 671$ and $1: 387$ (average 1:529), respectively (Table 1 ). Furthermore, higher levels of long-lasting antivaccinia Nabs were consistently found in the i.n. and i.o. groups when compared with the i.m. and s.c. groups (Figure $1(\mathrm{c})$ ). The average $\mathrm{IC}_{50}$ value of mucosal groups (i.n. and i.o.) is about 4.4-fold higher than that of i.m. and s.c routes $(1: 2331$ versus $1: 529, P<.01)$. Randomly selected individualmice of each group were tested with consistent results obtained.
3.3. Protection against Homologous Routes of Mucosal MVTT$S$ Challenges. To determine the long-lasting efficacy of the four routes of VTT vaccination in parallel, ten groups of vaccinated mice were challenged with MVTT-S six months after the vaccination. Each mouse received $2 \times 10^{6} \mathrm{PFU}$ of MVTT-S, the highest possible dose based on our viral stocks, via the i.n. or i.o. routes, respectively, which mimic the natural transmission of the variola virus. Since no anti$S$ antibodies were detected three weeks after challenge in all groups, we subsequently gave another MVTT-S challenge at the same dose in the same way. We found that i.o. and i.n. VTT vaccinations protected mice significantly against the homologous i.o. and i.n. MVTT-S challenges, respectively, without anti-S antibodies detected after the second challenge (Figure 2(a) and Table 1). The lack of seroconversion to $\mathrm{S}$ serves as a biomarker of the protection against MVTT-S infection.

\subsection{Protection against Alternating Routes of Mucosal MVTT-} $S$ Challenges. We then analyzed other groups of animals. We found that i.o. and i.n. VTT vaccinations protected mice completely against heterologous routes of i.n. and i.o. MVTT-S challenges, respectively, without any detectable anti-S Nabs (Figure 2(a)). In contrast, MVTT-S challenges resulted in high levels of anti-S Nabs in VTT-naïve or nonvaccinated mice after the second challenge (Figure 2(a)). The $\mathrm{IC}_{50}$ values reached over $1: 10,000$ serum dilution titer. Moreover, i.m. VTT vaccination also protected mice completely against i.n. and i.o. MVTT-S challenges, respectively (Figure 2(a)). Here, the lack of detectable anti-S Nabs after two consecutive MVTT-S challenges likely suggests a possible complete protection (Figure 2(a)). Notably, the s.c. VTT vaccination did not completely protect mice against i.n. or i.o. MVTT-S challenges as anti-S antibodies were readily detected three weeks after the second challenge (Figure 2(b)). Since the mean anti-S $\mathrm{IC}_{50}$ titer of all s.c. vaccinated mice was significantly lower than that of all nonvaccinated controls $(1: 1778$ versus $1: 14678, P=.03)$ (Figures $2(a)$ and $2(b)$ ), we speculated that at least partial significant protection was achieved by the s.c. VTT vaccination. This $P=.03$ value was determined by comparing the anti-S mean value of the s.c. vaccinated group $(1: 1598,1: 1026$, and $1: 2710$, with an average value of $1: 1778)$ versus control group $(1: 14261$, $1: 8251$, and $1: 21523$, with an average value of $1: 14678$ ). It is possible that the less protection of s.c. vaccinated mice is related to lower levels of anti-VTT neutralizing antibodies induced (Figure 1(a) and Table 1). Since MVTT-S causes transient viremia and infection in mice as we recently described [19], our efforts to detect live challenge virus around three weeks after each challenge in all groups were unsuccessful.

3.5. Lack of Complete Protection against Intramuscular MVTT-S Challenges. Since mucosal VTT vaccination induced likely full protection against homologous and heterologous routes of mucosal MVTT-S challenges (Figure 2(a)), we sought to determine whether the protection would apply to a systemic route of viral challenge. For this 


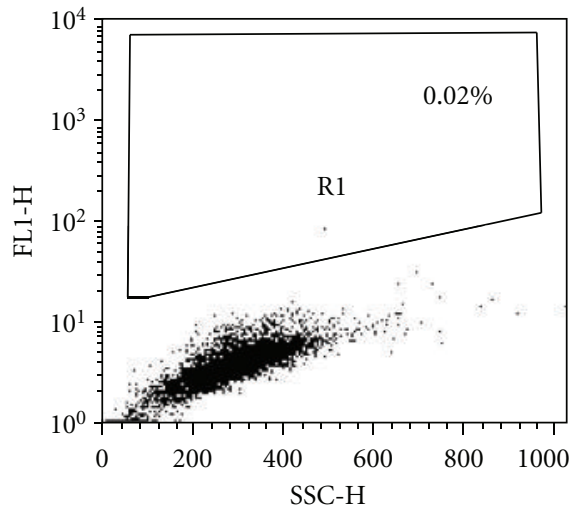

Negative control: uninfected cells

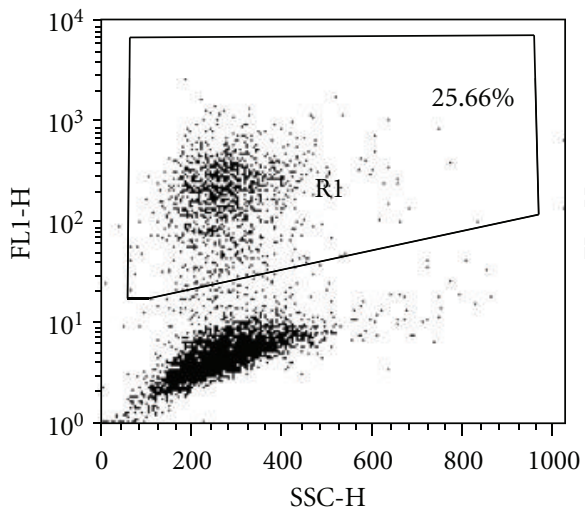

MVTT-GFP-infected cells

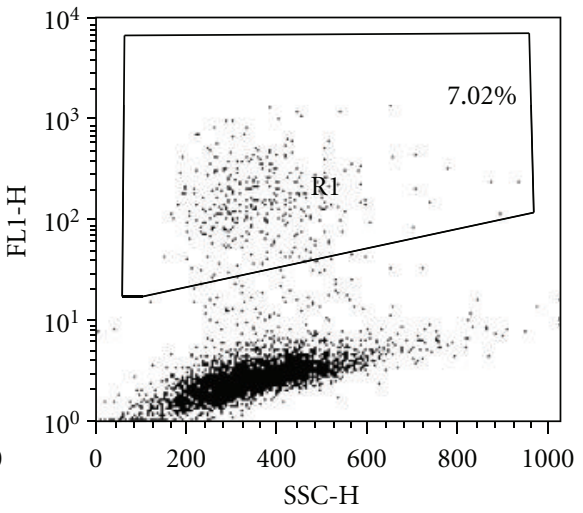

Neutralization of MVTT-GFP

(a)

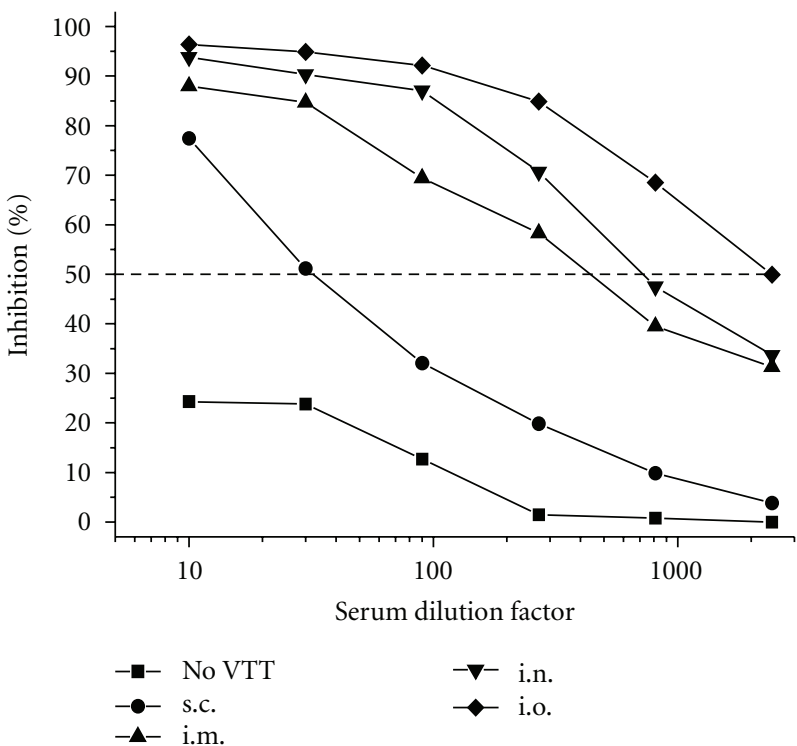

(b)

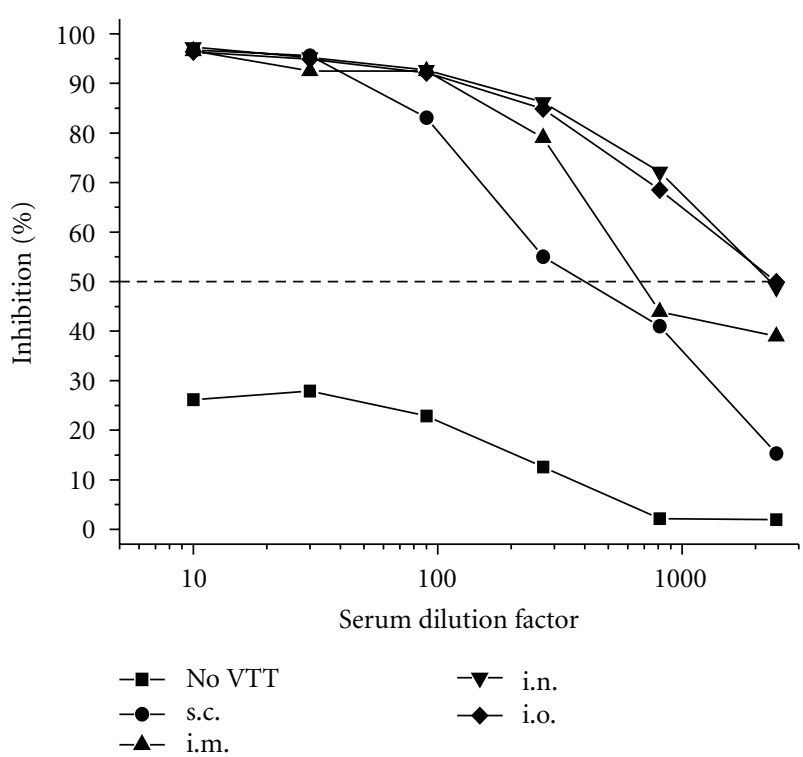

(c)

FIGURE 1: Anti-VTT neutralizing antibody responses after the VTT vaccination. (a) A FACS-based neutralization assay was newly developed to measure the anti-VTT neutralizing antibody responses. (b) Serum samples were tested three weeks after the VTT vaccination. (c) Serum samples were tested six months after the VTT vaccination. Due to a limited amount of serum collected from each mouse, pooled sera, which contain an equal amount of serum of each vaccinated mouse in each group, were subjected to each experiment. The $X$-axis represents the serum dilution factor whereas the $Y$-axis indicates the percentage of viral inhibition. $A n \mathrm{IC}_{50}$ value is a serum dilution factor which achieves $50 \%$ of viral inhibition as indicated by the dashed horizontal line. The experiment was repeated twice with similar results obtained. Placebo (no VTT) mice were tested as well.

TABLE 1: Neutralizing antibody titer from different time points after VTT vaccination and MVTT-S Challenges.

\begin{tabular}{|c|c|c|c|c|c|c|c|c|c|c|}
\hline Groups & \multicolumn{2}{|c|}{ i.m. } & \multicolumn{2}{|c|}{ i.n. } & \multicolumn{2}{|c|}{ i.o. } & \multicolumn{2}{|c|}{ s.c. } & \multicolumn{2}{|c|}{ placebo } \\
\hline \multicolumn{11}{|l|}{ Vaccination } \\
\hline VTT (3 weeks) & NA & 428 & NA & 711 & NA & 2370 & NA & 32 & NA & ND \\
\hline VTT (6 months) & NA & 671 & NA & 2265 & NA & 2397 & NA & 387 & NA & ND \\
\hline \multicolumn{11}{|l|}{ MVTT-S Challenge } \\
\hline i.m. (1st) & ND & 3288 & ND & 5249 & ND & 4742 & ND & 333 & ND & 46 \\
\hline i.m. (2nd) & 2732 & 6280 & 9369 & 5210 & 936 & 4721 & 1598 & 1386 & 14261 & 718 \\
\hline i.n. (1st) & ND & 2065 & ND & 5141 & ND & 6838 & ND & 520 & ND & 349 \\
\hline i.n. (2nd) & ND & 1587 & ND & 2944 & ND & 6205 & 1026 & 350 & 8251 & 1004 \\
\hline i.o. (1st) & ND & 4555 & ND & 4937 & ND & 2601 & ND & 35 & ND & 101 \\
\hline i.o. (2nd) & ND & 2362 & ND & 5148 & ND & 4901 & 2710 & 221 & 21523 & 1014 \\
\hline
\end{tabular}

The values of anti-S IC 50 are in bold whereas the values of anti-VTT IC 50 are not. NA: not applied; ND: not detected. 


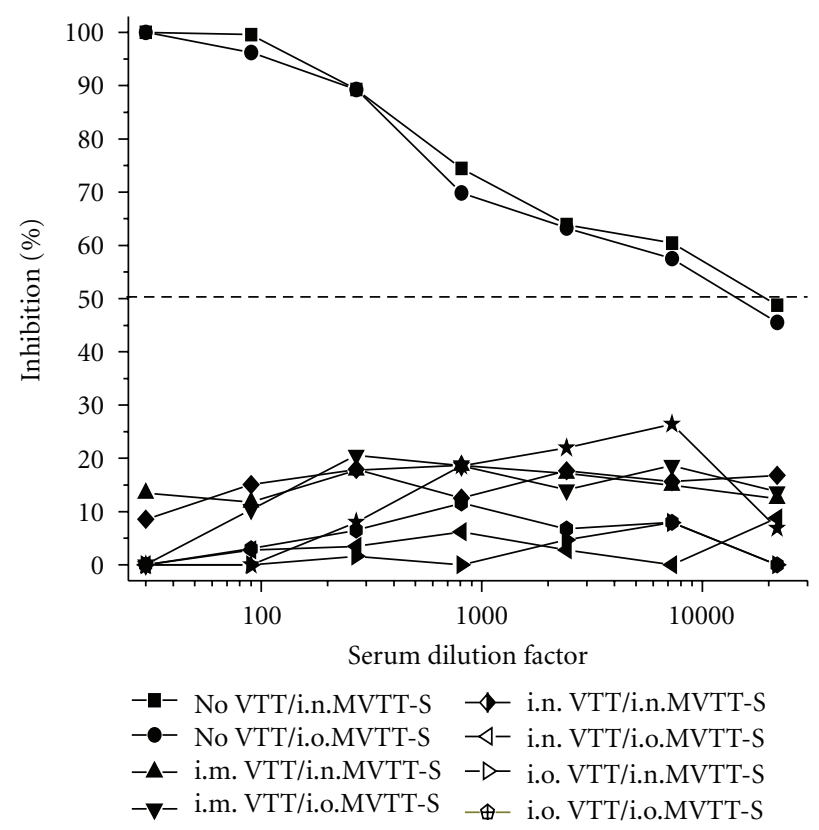

(a)

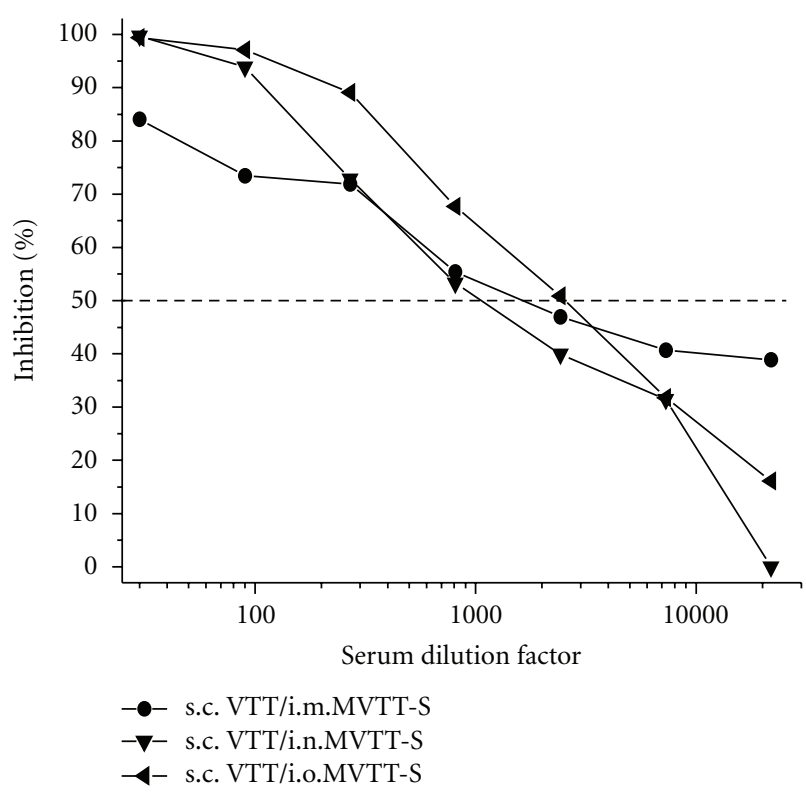

(b)

FIGURE 2: Anti-S neutralizing antibody responses in VTT-vaccinated mice after two MVTT-S challenges. (a) Serum samples of i.m., i.n., and i.o. VTT-vaccinated as well as of placebo-inoculated (no VTT) mice were tested. (b) Serum samples of s.c. VTT-vaccinated mice were tested. Pooled sera, which contain an equal amount of serum of each vaccinated mouse in each group, were subjected to each experiment. The $X$-axis represents the serum dilution factor whereas the $Y$-axis indicated the percentage of viral inhibition. The dashed horizontal line indicates $\mathrm{IC}_{50}$ values. The experiment was repeated twice with similar results obtained.

purpose, another three groups of VTT-vaccinated mice were challenged with MVTT-S via the i.m. route also at six months postvaccination. Similarly, no detectable levels of anti-S Nabs were found three weeks after the first MVTT$S$ challenge, suggesting a protective role of VTT-induced immunity. However, we found that the second MVTT-S challenge was able to generate substantial levels of anti-S Nabs in all three groups (Figure 3). Since the levels of anti$\mathrm{S}$ Nabs (both $\mathrm{IC}_{90}$ and $\mathrm{IC}_{50}$ values) were lower in three vaccinated groups compared to the placebo nonvaccinated group, it is likely that i.m., i.n., and i.o. vaccination conferred partial significant protection against MVTT-S challenges. For example, the serum dilution factors based on the $\mathrm{IC}_{50}$ value via different routes of prevaccination (placebo $>$ i.n., i.o., or i.m.) were $1: 14261>1: 9369,1: 936$, or $1: 2732$, respectively (Table 1 and Figure 3).

Since substantial levels of anti-VTT Nab responses were induced by a one time VTT vaccination via four different routes (Figure 1(b)), it was puzzling why these Nabs were not able to block i.m. MVTT-S infections completely. For this reason, we speculated that there might be a difference in the recalled anti-VTT Nab responses after the MVTT$S$ challenges. Therefore, we measured the levels of antiVTT Nab responses after each of the two MVTT-S challenges. We found that the first MVTT-S challenge boosted the levels of anti-VTT Nabs significantly, confirming its shared neutralizing determinants with VTT (Figure 4(a)). The average serum dilution factor of antivaccinia Nabs among i.m., i.n., and i.o. VTT-vaccinated mice had reached

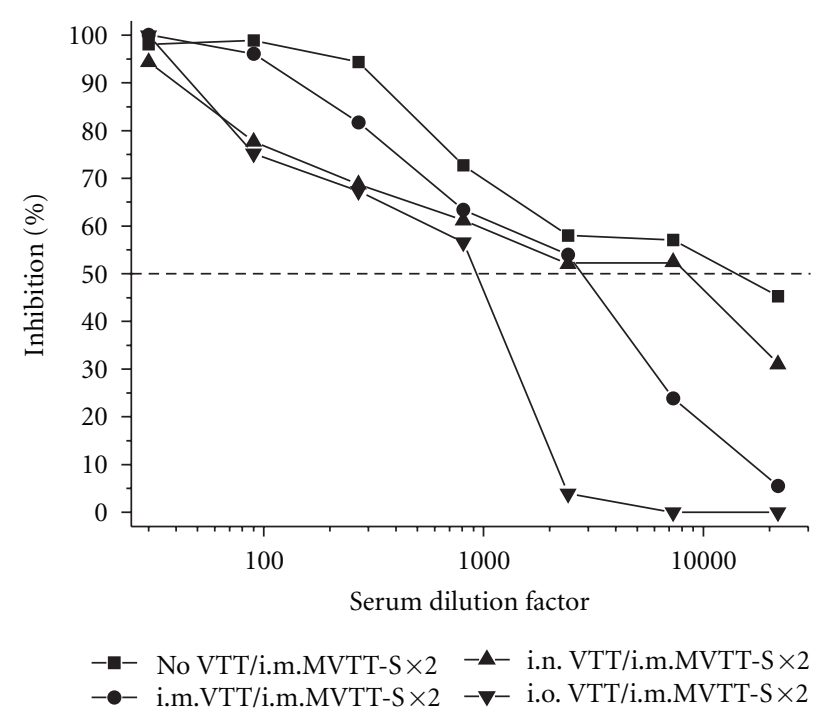

FIgUre 3: Anti-S neutralizing antibody responses in VTT-vaccinated mice after two i.m. MVTT-S challenges. Serum samples collected three weeks after the second MVTT-S challenge were tested in this experiment. Pooled sera, which contain an equal amount of serum of each vaccinated mouse in each group, were subjected to each experiment. The $X$-axis represents the serum dilution factor whereas the $Y$-axis indicated the percentage of viral inhibition. The dashed horizontal line indicates $\mathrm{IC}_{50}$ values. The experiment was repeated twice with similar results obtained. As controls, no VTT represents mice, who did not receive VTT-vaccination. 


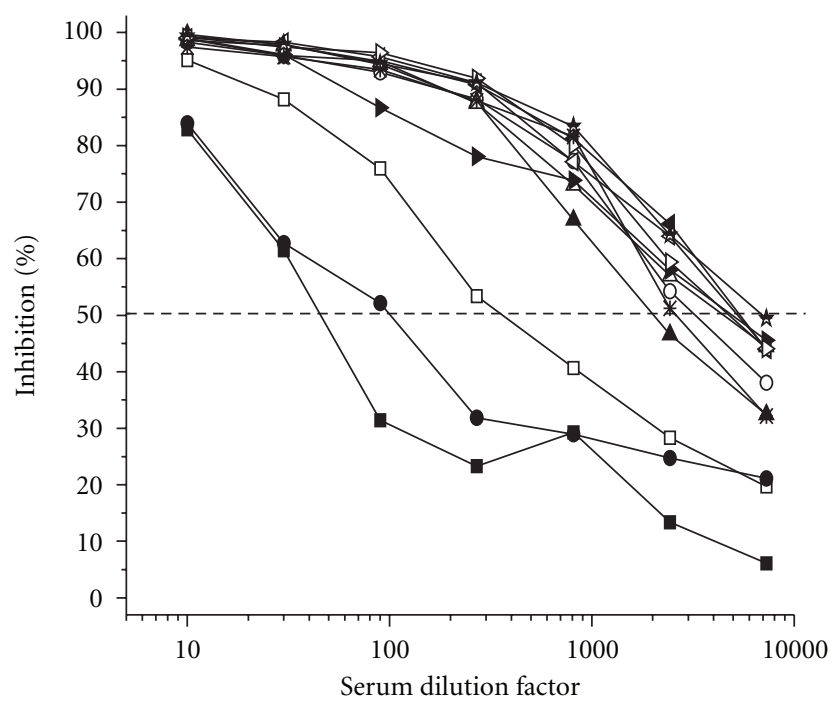

(a)

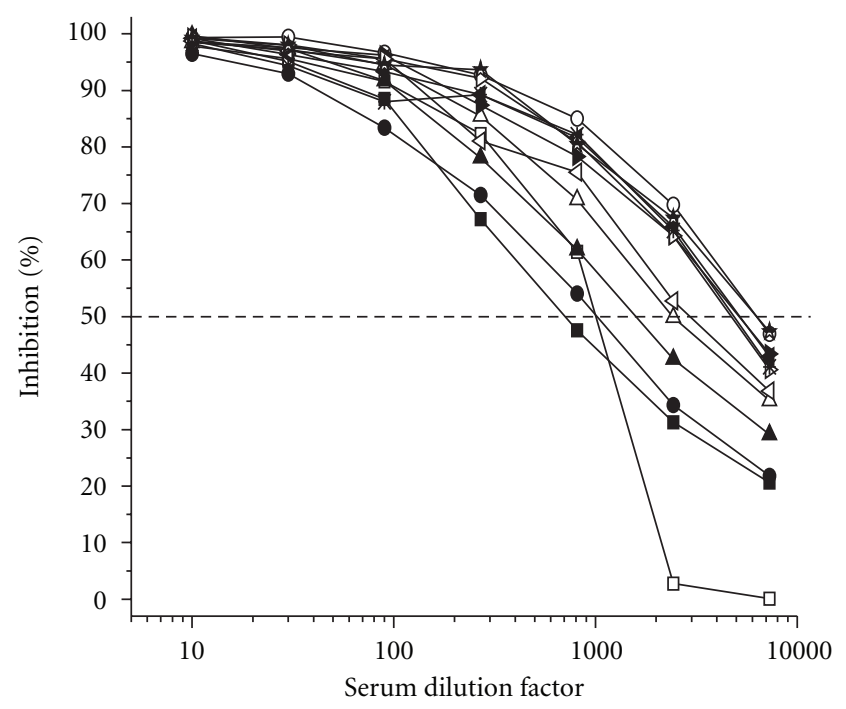

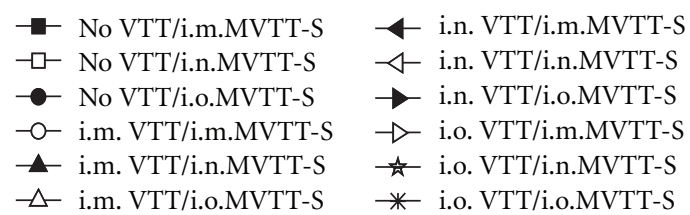

(b)

Figure 4: Anti-VTT neutralizing antibody responses after the first (a) and the second (b) MVTT-S challenges. Serum samples collected three weeks after each of the two MVTT-S challenges were included in this experiment. Pooled sera, which contain an equal amount of serum of each vaccinated mouse in each group, were subjected to each experiment. The $X$-axis represents the serum dilution factor whereas the $Y$-axis indicates the percentage of viral inhibition. The dashed horizontal line indicates $\mathrm{IC}_{50}$ values. The experiment was repeated twice with similar results obtained. As controls, no VTT represents mice, who did not receive VTT-vaccination. comparable levels with $\mathrm{IC}_{50}$ values of $1: 3303,1: 5109$, and $1: 4727$, respectively, which is not statistically significant (Figure $4(\mathrm{a})$ ). This $P$ value was calculated from the average values of anti-VTT Nabs after the first challenge from three groups: i.m. $(1: 3288,1: 2065$, and $1: 4555$, with an average of $1: 3303)$, i.n. $(1: 5249,1: 5141$, and $1: 4937$, with an average of $1: 5109)$, and i.o. (1:4742, $1: 6838,1: 2601$, with an average of $1: 4727)$. In addition, we determined the antiVTT Nab response after the second challenge of MVTTS. We found that the average serum dilution titer of antiVTT Nabs among i.m., i.n., and i.o. pre-VTT-vaccinated mice had likely saturated at comparable levels with the $\mathrm{IC}_{50}$ values of $1: 3410,1: 4434$, and $1: 5276$, respectively, which is not statistically significant (Figure $4(\mathrm{~b})$ ). This $P$ value was calculated from the average values of anti-VTT Nabs after the second challenge from three groups: i.m. (1:6280, $1: 1587$, and $1: 2362$, with an average of $1: 3410)$, i.n. (1:5210, $1: 2944$, and $1: 5148$, with an average of $1: 4434)$, and i.o. $(1: 4721,1: 6205$, and $1: 4901$, with an average of $1: 5276)$. Apparently, these levels of the recalled anti-VTT Nabs were sufficient to block completely the mucosal (i.n. and i.o.) but not the systemic (i.m.) MVTT-S challenges, suggesting a likely difference between mucosal and systemic immunity.

\section{Discussion}

The smallpox vaccination, which involves live vaccinia virus, remains a gold standard of vaccines because it has led to the complete eradication of a deadly human viral disease. To our knowledge, this study is the first to provide a parallel experimental comparison of four different routes of VTT vaccination for inducing protective efficacy in a mouse model. We provide evidence that one-time vaccination with VTT through each of four different routes was able to induce long-lasting antivaccinia $\mathrm{Nab}$ responses in mice. Apparently, higher levels (4.4-fold) of the Nab responses were induced via the mucosal i.n. and i.o. inoculations when compared with the i.m. and s.c. routes. Moreover, the i.n. and i.o. VTT vaccination likely protected animals completely from two consecutive mucosal challenges of the MVTT-S strain via either homologous (i.n. and i.o.) or heterologous (i.o. and i.n.) routes, respectively, (Figure 2(a)). As controls, s.c. VTT vaccination did not achieve full protection against i.m., i.n., or i.o. MVTT-S challenges, respectively, (Figure 2(b)). While i.m. VTT vaccination fully protected animals from i.n. and i.o. MVTT-S challenges, i.m., i.n., and i.o. VTT-vaccinated animals were not fully protected from i.m. challenges (Figures 2(a) and 3). Our results, therefore, suggested that the route of VTT vaccination is critical for inducing protection in mice. In the meantime, the finding that i.m. MVTT$S$ challenge overcomes preexisting antivaccinia immunity induced via mucosal routes has implications for MVTTbased mucosal vaccine development.

The route of VTT vaccination determines the level of long-lasting antivaccinia Nabs induced. It has been demonstrated that Nabs induced by vaccinia vaccine play an essential role in providing sterile immunity against smallpox infections $[25,26]$. Here we show that VTT displays an advantage for inducing higher levels of antivaccinia Nabs 
through the noninvasive mucosal route of vaccination. The underlying mechanism of this finding remains largely unknown, although we have recently provided evidence that the tissue tropism may play a role in affecting the immunogenicity of a vaccinia-based vaccine [19]. Since VTT and its variants unlikely establish long-term persistent infection in mice, mechanisms for the maintenance of immunological memory for lasting Nabs remain to be further investigated [8]. One study showed that antigen can be retained in the draining lymph nodes for extended periods of time [27]. Whether this finding would have implications for the continuous maturation of VTT-induced Nabs observed in our study will require future studies. We acknowledge that different route of vaccination may have different protective effects. We did not conduct a direct comparison between intradermal (i.d.) vaccination with other routes because i.d. vaccination did not seem to induce high levels of Nabs in our previous studies [17]. We, however, will compare the skin scarification vaccination with other routes in our future studies as it conferred sterile protection in humans against smallpox infection historically.

Traditional VV challenge models involved the use of highly pathogenic and lethal dose viruses for challenges, which require special biosafety containments. In this study, we developed a convenient model for animal challenge based on testing of the antibody response to the highly immunogenic S protein. Through our protocol, the preparation of vaccinia virus was mostly intracellular mature virion (IMV) particles. Since the $S$ spike protein was not located on the IMV by Western blot analysis (data not shown), the MVTT-S particles themselves cannot induce S-specific antibodies unless they enter the cells and initiate infection. This design provides anti-S antibody as a valuable biological indicator for protection of MVTT-S challenge in mice and eliminates the biosafety issues related to the restricted use of pathogenic vaccinia WR by laboratory regulations [28], as well as helps to reduce study costs. Nonpathogenic viral challenge model has previously been used to study the neutralizing antibody-mediated protection against immunodeficiency viral infection [29]. We, however, recognize the limitation of our experimental system because there might be major differences between lethal and nonpathogenic vaccinia challenge systems. For example, NKT-cells, CD8+ CTLs together with Nabs may be involved in protection against a highly pathogenic virus [30]. This issue should be further studied in future studies.

Mucosal VTT vaccination elicited protection against both homologous and heterologous routes of mucosal MVTT-S challenges. Our results have provided scientific evidence that mucosal VTT vaccination (e.g., one time i.n. or i.o. VTT vaccination in this study) likely confers complete protection against mucosal routes of viral challenges (Figure 2(a)), which is consistent to previous findings $[14,31]$. These findings are relevant to smallpox prevention because the vaccination likely provided a protective barrier at the dominant portal of variola viral entry, in the mucosa. This is concluded by the fact that the transmission of smallpox occurs mainly through inhalation of airborne variola virus, usually droplets expressed from the oral, nasal, or pharyngeal mucosa of an infected person [32]. Critically, the role of mucosal immunization with a smallpox vaccine has been demonstrated in previous studies [33, 34]. Since four groups of mice all developed systemic antivaccinia Nabs before MVTT-S challenges (Figure 1(c)), we speculated that higher levels (4.4-fold) of the Nab responses induced via the mucosal i.n. and i.o. inoculations compared with the s.c. route might have played a role in blocking MVTT-S challenge completely. To this end, the rapidly recalled high levels of anti-VTT Nabs after the MVTT-S challenges might also contribute to this protection (Figure 4(a)). In spite of these findings, extra attentions should be paid when VTT is used as a human smallpox vaccine for mucosal vaccination due to (1) its in vivo toxicity or virulence [16] as well as (2) its complicated quasispecies containing a group of viral variants [14]. As a substitute, it is evident to use a safely attenuated and homogenous MVTT strain to conduct mucosal smallpox vaccinations [14].

One obstacle of the application of vaccinia-based vaccine is the preexisting antivector immunity [35]. This is often an issue for the clinical development of a vacciniabased vaccine. Previous studies indicated that mucosal vaccination overcomes preexisting antivaccinia immunity generated through skin vaccination $[35,36]$. Consistently, we showed that i.n. or i.o. MVTT-S challenges overcame preexisting antivaccinia immunity generated through s.c. VTT vaccination for inducing anti-S Nabs (Figure 2(b)) [17]. Moreover, we now provide a new finding that i.m. MVTT$S$ challenges overcome preexisting antivaccinia immunity generated through s.c., i.n., or i.o. route of VTT vaccination (Figures 2(a) and 3). Our findings have implications either for overcoming the preexisting antivaccinia immunity or for improving the immunogenicity of a vaccinia-based vaccine. Since vaccinia-based vaccines have been developed for inducing protective immune responses at the mucosal sites of viral transmission for HIV and other pathogens [3739], our findings provide evidence to support a new strategy of heterologous routes of prime and boost vaccination using the same vaccine. In comparison to heterologous prime and boost regimens using different viral vectors [40, 41], this is an area of interest for further investigations.

One limitation of our study is the lack of measurements of T-cell-mediated immunity. Besides Nabs, it is possible that the observed VTT-induced protection was partially contributed by cell-mediated immunity. To this end, the mucosal route of vaccination may also be crucial for the generation of highly protective T-cell-mediated immunity. A recent study demonstrated that protection against lethal respiratory vaccinia challenge requires both respiratory mucosal $\mathrm{T}_{\mathrm{EM}}$ cells and central memory $\mathrm{T}$ cells [42]. A future study is, therefore, necessary to address the protective effects of specific cell-mediated immunity under the similar experimental conditions.

\section{Acknowledgments}

This work was supported by the Chinese 973 project 2006CB504208, the 11th 5-year project 2008ZX10001-011 and 2008ZX10001-015, Hong Kong research grant council 
(HK-RGC762209 to ZC), and the UDF/LKS grants of the University of Hong Kong to its AIDS Institute. We thank Jenny $\mathrm{Ng}$ for editorial inputs. B. Lu, W. Yu, and X. Huang contributed equally to this work.

\section{References}

[1] V. A. Fulginiti, A. Papier, J. M. Lane, J. M. Neff, and D. A. Henderson, "Smallpox vaccination: a review, part II. Adverse events," Clinical Infectious Diseases, vol. 37, no. 2, pp. 251-271, 2003.

[2] S. D. Nafziger, "Smallpox," Critical Care Clinics, vol. 21, no. 4, pp. 739-746, 2005.

[3] D. A. Henderson, "The eradication of smallpox," Scientific American, vol. 235, no. 4, pp. 25-33, 1976.

[4] M. A. Sauri, J. A. Frelinger, M. L. Garba, R. B. Belshe, and S. E. Frey, "Responses to smallpox vaccine," The New England Journal of Medicine, vol. 347, no. 9, pp. 689-690, 2002.

[5] D. L. Heymann, "Smallpox containment updated: considerations for the 21st century," International Journal of Infectious Diseases, vol. 8, supplement 2, pp. S15-S20, 2004.

[6] I. J. Amanna, I. Messaoudi, and M. K. Slifka, "Protective immunity following vaccination: how is it defined?" Human Vaccines, vol. 4, no. 4, pp. 316-319, 2008.

[7] I. J. Amanna, N. E. Carlson, and M. K. Slifka, "Duration of humoral immunity to common viral and vaccine antigens," The New England Journal of Medicine, vol. 357, no. 19, pp. 1903-1915, 2007.

[8] I. J. Amanna, M. K. Slifka, and S. Crotty, "Immunity and immunological memory following smallpox vaccination," Immunological Reviews, vol. 211, pp. 320-337, 2006.

[9] S. Mahalingam, I. K. Damon, and B. A. Lidbury, "25 years since the eradication of smallpox: why poxvirus research is still relevant," Trends in Immunology, vol. 25, no. 12, pp. 636-639, 2004.

[10] R. Weltzin, J. Liu, K. V. Pugachev et al., "Clonal vaccinia virus grown in cell culture as a new smallpox vaccine," Nature Medicine, vol. 9, no. 9, pp. 1125-1130, 2003.

[11] X. Tang and Z. Chen, "The development of an AIDS mucosal vaccine," Viruses, vol. 1, no. 2, pp. 283-297, 2010.

[12] F. Fenner, D. A. Henderson, I. Arita, Z. Jezek, and I. D. Ladnyi, Smallpox and Its Eradication, World Health Organization, Geneva, Switzerland, 1988.

[13] S. L. Dong, Q. C., "The founder of vaccinia virus Tiantan strain," Weishengwuxue Mianyixue Jinzhan, vol. 37, no. 3, pp. $1-3,2009$.

[14] W. Yu, Q. Fang, W. Zhu et al., "One time intranasal vaccination with a modified vaccinia Tiantan strain MVTT(ZCI) protects animals against pathogenic viral challenge," Vaccine, vol. 28, no. 9, pp. 2088-2096, 2010.

[15] M. Kretzschmar, J. Wallinga, P. Teunis, S. Xing, and R. Mikolajczyk, "Frequency of adverse events after vaccination with different vaccinia strains," PLoS Medicine, vol. 3, no. 8, article e272, 2006.

[16] Q. Fang, L. Yang, W. Zhu et al., "Host range, growth property, and virulence of the smallpox vaccine: vaccinia virus Tian Tan strain," Virology, vol. 335, no. 2, pp. 242-251, 2005.

[17] X. Huang, B. Lu, W. Yu et al., "A novel replication-competent vaccinia vector MVTT is superior to MVA for inducing high levels of neutralizing antibody via mucosal vaccination," PLoS One, vol. 4, no. 1, Article ID e4180, 2009.

[18] D. C. Tscharke, P. C. Reading, and G. L. Smith, "Dermal infection with vaccinia virus reveals roles for virus proteins not seen using other inoculation routes," Journal of General Virology, vol. 83, part 8, pp. 1977-1986, 2002.

[19] H. Liu, W. Yu, X. Tang et al., "The route of inoculation determines the tissue tropism of modified vaccinia Tiantan expressing the spike glycoprotein of SARS-CoV in mice," Journal of Medical Virology, vol. 82, no. 5, pp. 727-734, 2010.

[20] X. Huang, L. Liu, L. Ren, C. Qiu, Y. Wan, and J. Xu, "Mucosal priming with replicative Tiantan vaccinia and systemic boosting with DNA vaccine raised strong mucosal and systemic HIV-specific immune responses," Vaccine, vol. 25, no. 52, pp. 8874-8884, 2007.

[21] W. K. Joklik, "The purification of four strains of poxvirus," Virology, vol. 18, no. 1, pp. 9-18, 1962.

[22] Z. Chen, L. Zhang, C. Qin et al., "Recombinant modified vaccinia virus Ankara expressing the spike glycoprotein of severe acute respiratory syndrome coronavirus induces protective neutralizing antibodies primarily targeting the receptor binding region," Journal of Virology, vol. 79, no. 5, pp. 26782688, 2005.

[23] C. Tsai, C. Caillet, H. Hu et al., "Measurement of neutralizing antibody responses against $\mathrm{H} 5 \mathrm{~N} 1$ clades in immunized mice and ferrets using pseudotypes expressing influenza hemagglutinin and neuraminidase," Vaccine, vol. 27, no. 48, pp. 67776790, 2009.

[24] P. L. Earl, J. L. Americo, and B. Moss, "Development and use of a vaccinia virus neutralization assay based on flow cytometric detection of green fluorescent protein," Journal of Virology, vol. 77, no. 19, pp. 10684-10688, 2003.

[25] M. R. E. Benhnia, M. M. McCausland, H. P. Su et al., "Redundancy and plasticity of neutralizing antibody responses are cornerstone attributes of the human immune response to the smallpox vaccine," Journal of Virology, vol. 82, no. 7, pp. 37513768, 2008.

[26] Y. Edghill-Smith, H. Golding, J. Manischewitz et al., "Smallpox vaccine-induced antibodies are necessary and sufficient for protection against monkeypox virus," Nature Medicine, vol. 11, no. 7, pp. 740-747, 2005.

[27] N. Fazilleau, M. D. Eisenbraun, L. Malherbe et al., "Lymphoid reservoirs of antigen-specific memory T helper cells," Nature Immunology, vol. 8, no. 7, pp. 753-761, 2007.

[28] J. M. Neff, L. J. Michael, V. A. Fulginiti, and D. A. Henderson, "Contact vaccinia-transmission of vaccinia from smallpox vaccination," Journal of the American Medical Association, vol. 288, no. 15, pp. 1901-1905, 2002.

[29] H. L. Robinson, D. C. Montefiori, R. P. Johnson et al., "Neutralizing antibody-independent containment of immunodeficiency virus challenges by DNA priming and recombinant pox virus booster immunizations," Nature Medicine, vol. 5, no. 5, pp. 526-534, 1999.

[30] J. T. Snyder, I. M. Belyakov, A. Dzutsev, F. Lemonnier, and J. A. Berzofsky, "Protection against lethal vaccinia virus challenge in HLA-A2 transgenic mice by immunization with a single CD8+ T-cell peptide epitope of vaccinia and variola viruses," Journal of Virology, vol. 78, no. 13, pp. 7052-7060, 2004.

[31] W. Kastenmuller, G. Gasteiger, L. Stross, D. H. Busch, and I. Drexler, "Cutting edge: mucosal application of a lyophilized viral vector vaccine confers systemic and protective immunity toward intracellular pathogens," Journal of Immunology, vol. 182, no. 5, pp. 2573-2577, 2009.

[32] E. Elst, “The transmission mechanism of smallpox," Ärztliche Forschung, vol. 9, no. 1, pp. 13-32, 1955.

[33] I. M. Belyakov, D. Isakov, Q. Zhu, A. Dzutsev, D. Klinman, and J. A. Berzofsky, "Enhancement of CD8+ T cell immunity in the lung by $\mathrm{CpG}$ oligodeoxynucleotides increases protective 
efficacy of a modified vaccinia Ankara vaccine against lethal poxvirus infection even in a CD4-deficient host," Journal of Immunology, vol. 177, no. 9, pp. 6336-6343, 2006.

[34] I. M. Belyakov, P. Earl, A. Dzutsev et al., "Shared modes of protection against poxvirus infection by attenuated and conventional smallpox vaccine viruses," Proceedings of the National Academy of Sciences of the United States of America, vol. 100, no. 16, pp. 9458-9463, 2003.

[35] I. M. Belyakov, B. Moss, W. Strober, and J. A. Berzofsky, "Mucosal vaccination overcomes the barrier to recombinant vaccinia immunization caused by preexisting poxvirus immunity," Proceedings of the National Academy of Sciences of the United States of America, vol. 96, no. 8, pp. 4512-4517, 1999.

[36] T. Naito, Y. Kaneko, and D. Kozbor, "Oral vaccination with modified vaccinia virus Ankara attached covalently to TMPEG-modified cationic liposomes overcomes pre-existing poxvirus immunity from recombinant vaccinia immunization," Journal of General Virology, vol. 88, part 1, pp. 61-70, 2007.

[37] C. Ranasinghe, J. C. Medveczky, D. Woltring et al., "Evaluation of fowlpox-vaccinia virus prime-boost vaccine strategies for high-level mucosal and systemic immunity against HIV-1," Vaccine, vol. 24, no. 31-32, pp. 5881-5895, 2006.

[38] F. Martinon, P. Brochard, M. Ripaux et al., "Improved protection against simian immunodeficiency virus mucosal challenge in macaques primed with a DNA vaccine and boosted with the recombinant modified vaccinia virus Ankara and recombinant Semliki Forest virus," Vaccine, vol. 26, no. 4, pp. 532-545, 2008.

[39] N. P. Goonetilleke, H. McShane, C. M. Hannan, R. J. Anderson, R. H. Brookes, and A. V. S. Hill, "Enhanced immunogenicity and protective efficacy against Mycobacterium tuberculosis of bacille Calmette-Guerin vaccine using mucosal administration and boosting with a recombinant modified vaccinia virus Ankara," Journal of Immunology, vol. 171, no. 3, pp. 1602-1609, 2003.

[40] S. Lu, "Heterologous prime-boost vaccination," Current Opinion in Immunology, vol. 21, no. 3, pp. 346-351, 2009.

[41] L. Ba, C. E. Yi, L. Zhang, D. D. Ho, and Z. Chen, "Heterologous MVA-S prime Ad5-S boost regimen induces high and persistent levels of neutralizing antibody response against SARS coronavirus," Applied Microbiology and Biotechnology, vol. 76, no. 5, pp. 1131-1136, 2007.

[42] L. Liu, Q. Zhong, T. Tian, K. Dubin, S. K. Athale, and T. S. Kupper, "Epidermal injury and infection during poxvirus immunization is crucial for the generation of highly protective $\mathrm{T}$ cell-mediated immunity," Nature Medicine, vol. 16, no. 2, pp. 224-227, 2010. 


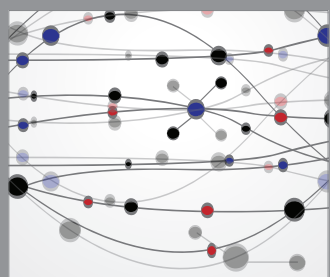

The Scientific World Journal
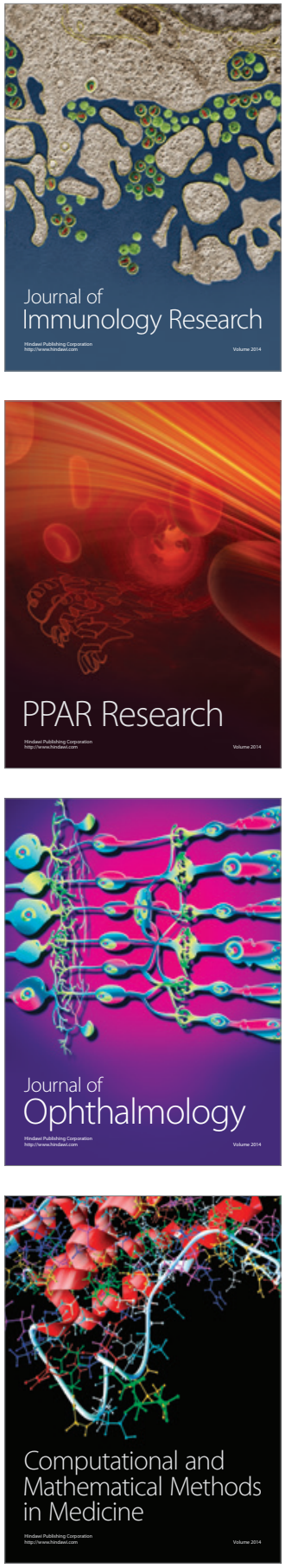

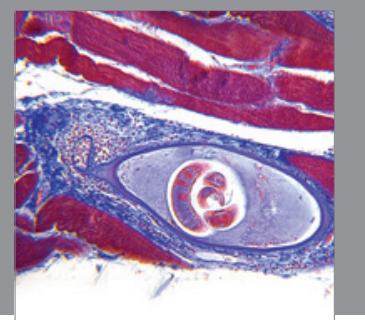

Gastroenterology

Research and Practice
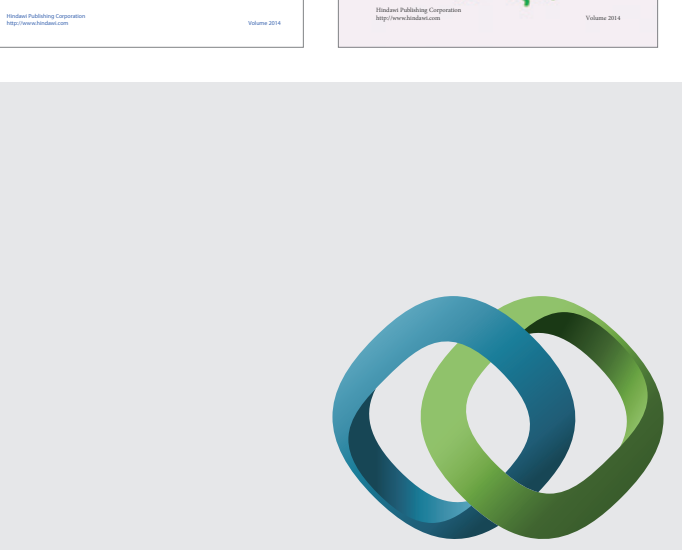

\section{Hindawi}

Submit your manuscripts at

http://www.hindawi.com
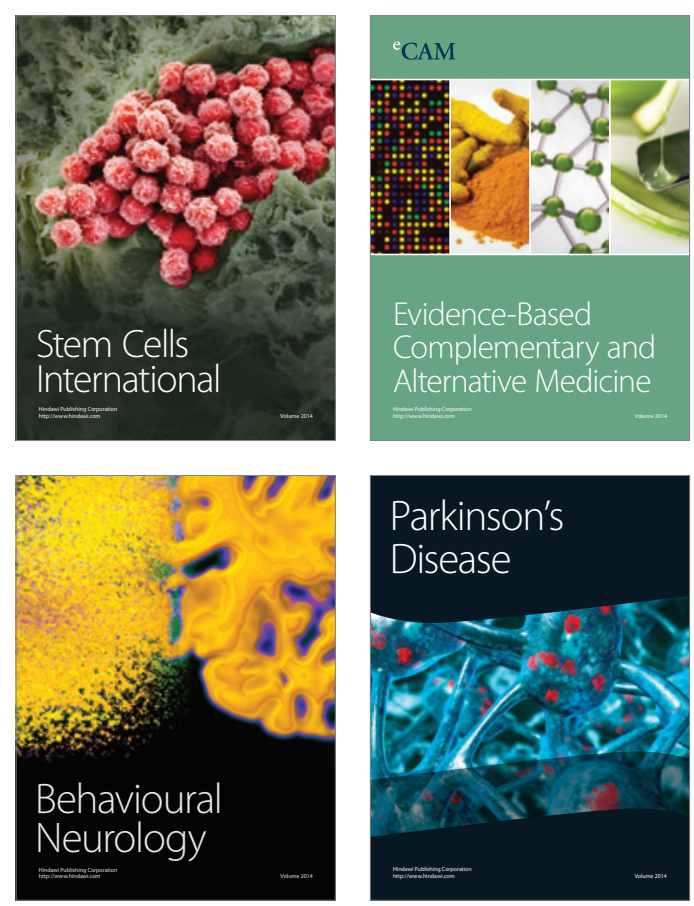

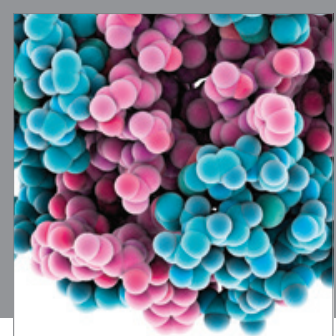

Journal of
Diabetes Research

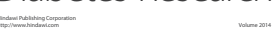

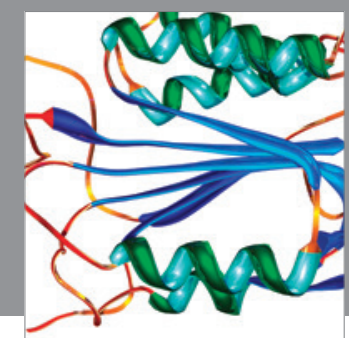

Disease Markers
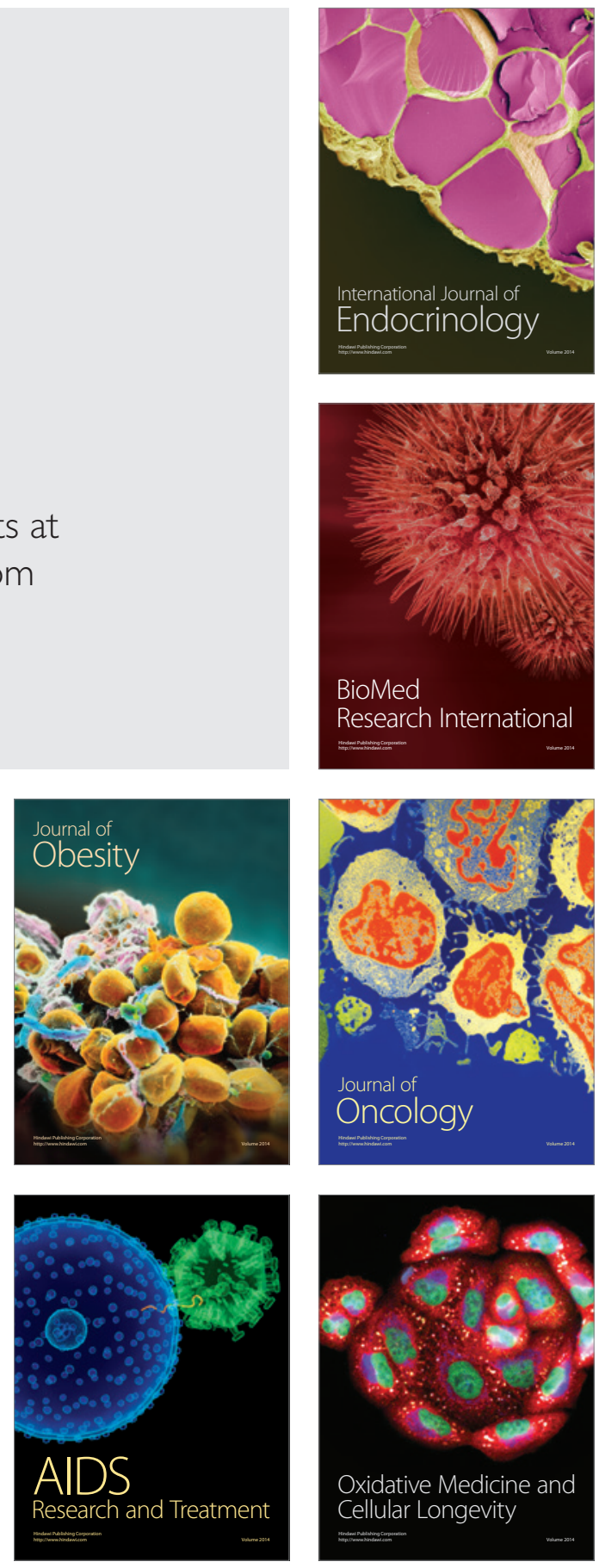